Dementia

and Geriatric

Cognitive Disorders
Dement Geriatr Cogn Disord 2017;43:281-293

DOI: 10.1159/000471761

Accepted: March 16, 2017

Published online: April 28, 2017
(C) 2017 S. Karger AG, Base

www.karger.com/dem

\title{
Pre-ESRD Dementia and Post-ESRD Mortality in a Large Cohort of Incident Dialysis Patients
}

\author{
Miklos Z. Molnar a, b Keiichi Sumida ${ }^{a}$ Abduzhappar Gaipova, c \\ Praveen K. Potukuchi ${ }^{a}$ Tibor Fülöp ${ }^{d, e}$ Kiran Joglekar ${ }^{f}$ Jun Ling Lu ${ }^{a}$ \\ Elani Strejag Kamyar Kalantar-Zadeh ${ }^{9}$ Csaba P. Kovesdy ${ }^{a}$ h \\ a Division of Nephrology, Department of Medicine, University of Tennessee Health Science \\ Center, Memphis, TN, USA; ${ }^{b}$ Department of Transplantation and Surgery, Semmelweis \\ University, Budapest, Hungary; ${ }^{\mathrm{C}}$ Department of Extracorporeal Hemocorrection, National \\ Scientific Medical Research Center, Astana, Kazakhstan; dFMC Extracorporeal Life Support \\ Center, Fresenius Medical Care, and ${ }^{\mathrm{e}}$ Medical and Health Science Center, University of \\ Debrecen, Debrecen, Hungary; ${ }^{f}$ Department of Medicine, University of Tennessee Health \\ Science Center, Memphis, TN, g Harold Simmons Center for Chronic Disease Research and \\ Epidemiology, Division of Nephrology and Hypertension, University of California-Irvine, \\ Orange, CA, and hephrology Section, Memphis VA Medical Center, Memphis, TN, USA
}

\section{Keywords}

End of life care, dementia - Mortality · Chronic kidney disease - End-stage renal disease · Transition

\begin{abstract}
Background: Conservative management may be a desirable option for elderly, fragile, or demented patients who reach end-stage renal disease (ESRD), yet some patients with dementia are placed on renal replacement therapy nonetheless. Methods: From a nationwide cohort of 45,076 US veterans who transitioned to ESRD over 4 contemporary years (October 1, 2007 to September 30, 2011), we identified 1,336 (3.0\%) patients with International Classification of Diseases, Ninth Revision, Clinical Modification code-based dementia diagnosis during the prelude (predialysis) period. We examined the association of prelude dementia with all-cause mortality within the first 6 months following transition to dialysis, using a propensity-matched cohort and Cox proportional hazards models. Results: In the entire cohort, the overall mean \pm standard deviation age at baseline was $72 \pm 11$ years, 95\% were male, $23 \%$ were AfricanAmerican, and $66 \%$ were diabetic. There were 8,080 (18.5\%) deaths (mortality rate, $412 ; 95 \%$ confidence interval [CI], 403-421/1,000 patient-years) in the dementia-negative group, and 396 (29.6\%) deaths (mortality rate, 708; 95\% CI, 642-782/1,000 patient-years) in the dementia-
\end{abstract}

Csaba P. Kovesdy, MD

Nephrology Section, Memphis VA Medical Center

1030 Jefferson Ave.

Memphis, TN 38104 (USA)

E-Mail ckovesdy@uthsc.edu 
Molnar et al.: Pre-ESRD Dementia and Post-ESRD Mortality in a Large Cohort of Incident Dialysis Patients

positive group in the entire cohort in the first 6 months after dialysis initiation. Presence of dementia was associated with higher risk of all-cause mortality (adjusted hazard ratio, 1.25; $95 \%$ CI 1.12-1.38) compared to dementia-free patients in the first 6 months after dialysis initiation. Conclusion: Pre-ESRD dementia is associated with increased risk of early post-ESRD mortality in veterans transitioning to dialysis.

(C) 2017 S. Karger AG, Basel

\section{Introduction}

Each year, over 100,000 Americans and a similar number of Europeans transition from very-late-stage non-dialysis-dependent chronic kidney disease (NDD-CKD) to renal replacement therapy (RRT) [1,2]. Transitioning of such substantial numbers of patients to dialysis or transplantation in the USA has led to a total end-stage renal disease (ESRD) expenditure of USD 41.2 billion annually, including USD 30.9 billion by the Medicare ESRD program alone [2]. Despite technological and pharmaceutical developments in the last decades, the mortality rate of patients on maintenance dialysis has remained high, especially in the early period after dialysis initiation [3, 4]. Recently, efforts have been made to identify potential factors contributing to or associated with higher mortality rates in patients with advanced NDD-CKD transitioning to RRT [3]. Some of these identified factors, such as older age, are not modifiable [3]; however, a majority of them are potentially modifiable, such as type of the vascular access [3, 5], functional dependence [6], presence of congestive heart failure, cancer [3], slope of residual kidney function [7], and adherence to medications [8].

In the mid-1990s, early mortality rates after transition to RRT were as high as $6 \%$ in the first 90 days and 32\% during the first dialysis year [4]. Although these rates have declined in the last decade, to date they still remain as high as $20 \%$ [3]. The main cause of death in this early period is cardiovascular death, but withdrawal from dialysis is responsible for $20 \%$ of deaths [3]. Sicker, more fragile elderly patients might have the highest rate of early transition mortality and early withdrawal from dialysis. The relatively high withdrawal rate and the very high early mortality rate bring up the question of whether dialysis is the ideal modality of choice for many patients, and which patients would have benefitted from remaining on conservative/palliative medical treatment rather than initiating invasive dialysis therapy. Most recently, the Dialysis Advisory Group of the American Society of Nephrology suggested that dialysis patients with a life expectancy of $<1$ year may desire a palliative approach to dialysis care, focusing on aligning patient treatment with patients' informed preferences [9]. Identifying factors contributing to higher early mortality rates and worse quality of life on dialysis would assist in clinical decision-making in consideration of deciding whether a patient should pursue conservative/palliative treatment or transition to dialysis. One important clinical characteristic to consider in this decision is dementia [10].

Dementia is a relatively frequent comorbidity in nursing home patients [11] as well as patients with NDD-CKD and ESRD. While dementia was detected in 5.1\% of individuals without CKD, the prevalence was 6.8\% in NDD-CKD individuals based on the third National Health and Nutrition Examination Survey (NHANES III) [12]. In patients on dialysis, the prevalence of dementia may be as high as $40 \%$ based on formal cognitive function studies [13]. In addition, the documented diagnosis of dementia in patients undergoing maintenance dialysis is associated with a 1.5- to 2-fold increase in mortality compared with individuals without dementia, based on the Dialysis Outcomes and Practice Patterns Study (DOPPS) [14] and data from the United States Renal Data System (USRDS) [15]. Comparable associations have been reported in earlier stages of NDD-CKD [12]. 
Molnar et al.: Pre-ESRD Dementia and Post-ESRD Mortality in a Large Cohort of Incident Dialysis Patients

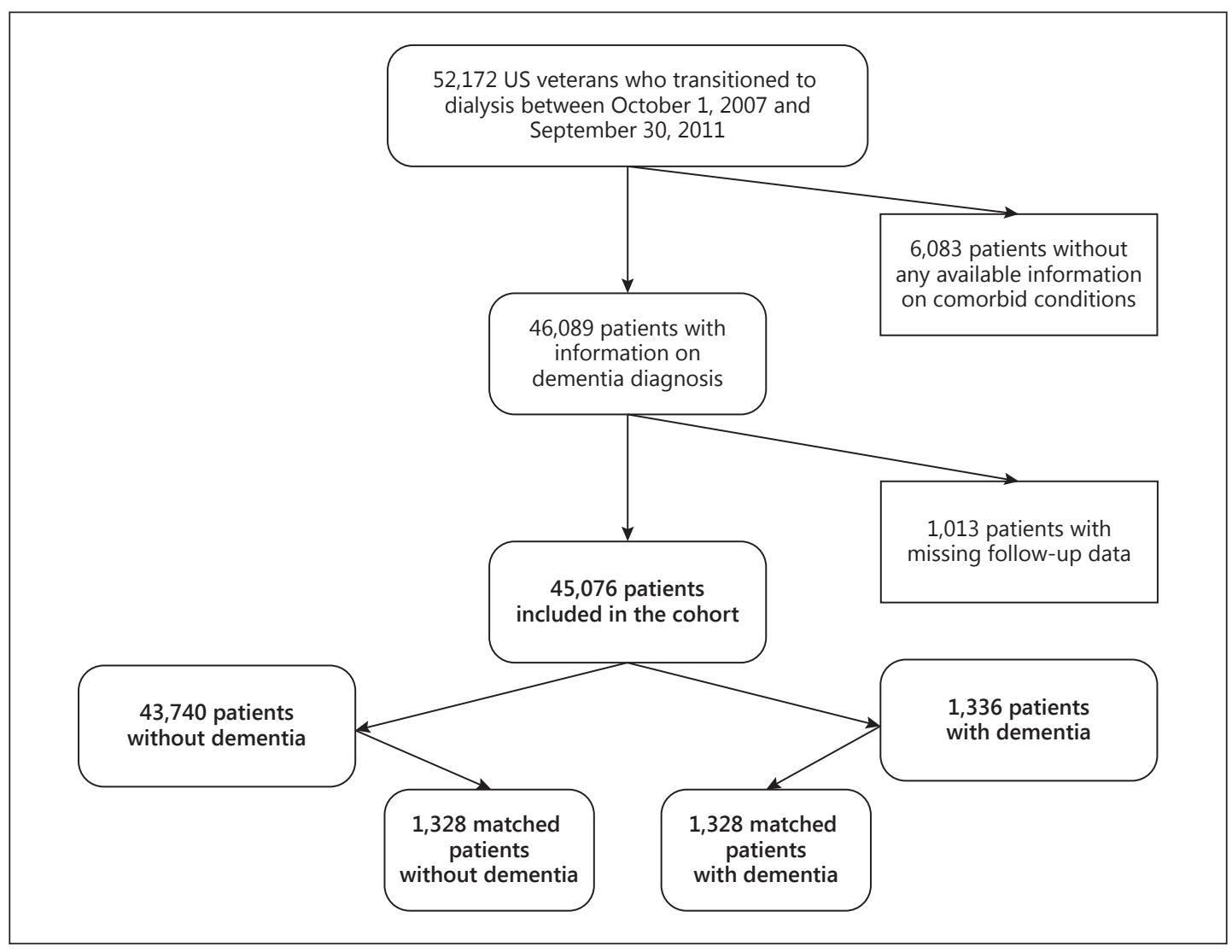

Fig. 1. Algorithm for the cohort definition.

However, prior studies assessing outcomes in dialysis patients with dementia examined prevalent ESRD patients, in whom dementia might have developed after the initiation of dialysis. Much less is known about the early post-ESRD outcomes associated with dementia in incident dialysis patients, in whom dementia diagnosed prior to dialysis transition could be a factor in deciding about starting dialysis. To address this knowledge gap, we aimed to investigate the association of dementia identified in the pre-ESRD transition period with post-ESRD early all-cause mortality, using a large nationally representative cohort of US veterans with advanced NDD-CKD transitioning to RRT. We hypothesized that presence of dementia in patients prior to transition is associated with a higher short- and long-term risk of death after transition to ESRD.

\section{Methods}

\section{Study Population}

We analyzed longitudinal data from the Transition of Care in CKD (TC-CKD) study, a retrospective cohort study examining US veterans with late-stage NDD-CKD transitioning to RRT from October 1, 2007 through September 30, 2011 [5, 8]. A total of 52,172 US veterans were identified from the USRDS [16] as a source population. The algorithm for the cohort definition is shown in Figure 1. We excluded patients without any available information on comorbid conditions $(n=6,083)$. We also excluded those with missing followup data ( $n=1,013$ who had died or received a kidney transplant on the date of transition to ESRD), resulting in a study population of 45,076 patients - 43,740 patients without dementia and 1,336 patients with dementia. Propensity score matching was applied, resulting in a cohort of 2,656 patients for survival analysis. 
Molnar et al.: Pre-ESRD Dementia and Post-ESRD Mortality in a Large Cohort of Incident Dialysis Patients

\section{Exposure Variable}

Dementia was defined as the presence of International Classification of Diseases, Ninth Revision, Clinical Modification (ICD-9-CM) codes for dementia (290.x, 294.1x, 331.2x) in any out- or inpatient medical record prior to transition to dialysis.

\section{Covariates}

Data from the USRDS Patient and Medical Evidence files were used to determine patients' baseline demographic characteristics at the time of dialysis initiation. Information on comorbidities at the time of dialysis initiation was extracted from VA Inpatient and Outpatient Medical SAS Datasets [17], using the ICD-9-CM diagnostic and Current Procedural Terminology codes, as well as from VA/Centers for Medicare and Medicaid Services (CMS) data. Medication data were collected from both CMS data (Medicare Part D) and VA pharmacy dispensation records [18]. Patients who received at least 1 dispensation of medications within the 6-month prelude period immediately preceding ESRD transition were recorded as having been treated with these medications. Laboratory data were obtained from VA research databases as previously described $[19,20]$, and their baseline values were defined as the average of each covariate during the 6-month prelude period preceding dialysis initiation. Estimated glomerular filtration rate (eGFR) was calculated using the Chronic Kidney Disease Epidemiology Collaboration equation [21].

\section{Outcome Assessment}

The primary outcome of interest was all-cause mortality after dialysis initiation. All-cause mortality data, censoring events, and associated dates were obtained from VA and USRDS data sources [7]. The start of the follow-up period was the date of dialysis initiation, and patients were followed up until death or other censoring events, including kidney transplantation, loss to follow-up, or end of follow-up period (6 and 12 months after dialysis initiation or December 27, 2012 for the entire follow-up period) [5, 8]. The primary analysis used a follow-up time of 6 months, as we wanted to focus on short-term post-dialysis mortality.

\section{Statistical Analysis}

Baseline patient characteristics were summarized according to the presence or absence of dementia prior to ESRD, and presented as percentage for categorical variables and mean \pm standard deviation (SD) for continuous variables. Differences between categories were assessed using standardized differences before and after propensity score matching.

Predictors of presence of dementia were assessed using logistic regression analyses. The propensity score method was used to account for baseline differences arising from dissimilarities in clinical and demographic characteristics of patients with and without dementia. Variables associated with the presence of dementia were identified using logistic regression and were used to calculate propensity scores. Stata's psmatch2 command suite was used to generate the propensity-matched cohorts by a 1-to-1 nearest neighbor matching without replacement. The following variables were included in the logistic regression model to create the propensity score: age, gender, race/ethnicity, marital status, comorbidities at baseline (myocardial infarction, congestive heart failure, peripheral vascular disease, cerebrovascular disease, paraplegia/hemiplegia, lung disease, rheumatic disease, peptic ulcer disease, HIV/AIDS, malignancy, liver disease, diabetes, hypertension), and medications at baseline (statins, vasodilators, renin-angiotensin-aldosterone system blockers, phosphate binders, erythropoiesis-stimulating agents, diuretics, calcium channel blockers, bicarbonates, $\beta$-blockers, active vitamin D).

The association between the presence of dementia and all-cause mortality was estimated using the Kaplan-Meier method and Cox proportional hazards models. All associations were examined in unadjusted models using our propensity-matched cohort of 2,656 patients.

We conducted several sensitivity analyses to evaluate the robustness of our main findings. To compare the effect of dementia on outcomes during different follow-up periods, we repeated our analyses separately using additional follow-up time definitions (i.e., the first 12 months after transition) as well as long-term follow-up (entire follow-up period up to 5 years). In addition, we also performed a sensitivity analysis using the propensity-matched cohort and further adjusted for serum albumin, blood hemoglobin, and eGFR. As the lack of these variables was substantial, the cohort in these analyses consisted of 1,840 patients. Moreover, we performed unadjusted and adjusted Cox regression models using the entire cohort population. Covariates in the adjusted models included age, gender, race/ethnicity, marital status, comorbidities at baseline (myocardial infarction, congestive heart failure, peripheral vascular disease, cerebrovascular disease, para- 
Molnar et al.: Pre-ESRD Dementia and Post-ESRD Mortality in a Large Cohort of Incident Dialysis Patients

Table 1. Patients' baseline characteristics before and after propensity score matching

\begin{tabular}{|c|c|c|c|c|c|c|}
\hline & \multicolumn{3}{|c|}{ Before propensity score matching } & \multicolumn{3}{|c|}{ After propensity score matching } \\
\hline & no dementia & dementia & std. diff. & no dementia & dementia & std. diff. \\
\hline Number of patients & 43,740 & 1,336 & $\mathrm{~N} / \mathrm{A}$ & 1,328 & 1,328 & $\mathrm{~N} / \mathrm{A}$ \\
\hline Age, years & $71.5 \pm 11.4$ & $77.0 \pm 9.2$ & 0.594 & $77.5 \pm 8.9$ & $77.0 \pm 9.2$ & 0.051 \\
\hline Male sex & $41,622(95.2)$ & $1,264(94.6)$ & -0.059 & $1,263(95.1)$ & $1,257(94.6)$ & -0.034 \\
\hline Race & & & -0.047 & & & -0.107 \\
\hline African-American & $10,099(23.1)$ & $367(27.5)$ & & $274(20.6)$ & $363(27.3)$ & \\
\hline White & $32,516(74.5)$ & $942(70.6)$ & & $1,003(75.5)$ & $941(70.8)$ & \\
\hline Other & $1,061(2.4)$ & $25(1.9)$ & & $51(3.8)$ & $24(1.8)$ & \\
\hline Marital status & & & 0.022 & & & -0.041 \\
\hline Married & $25,172(57.5)$ & $759(56.8)$ & & $824(62.0)$ & $756(56.9)$ & \\
\hline Divorced & $3,953(9.0)$ & $110(8.2)$ & & $84(6.3)$ & $109(8.2)$ & \\
\hline Single & $6,469(14.8)$ & $184(13.8)$ & & $145(10.9)$ & $184(13.8)$ & \\
\hline Widowed & $4,350(10.0)$ & $172(12.8)$ & & $165(12.4)$ & $170(12.8)$ & \\
\hline Other & $3,796(8.7)$ & $111(8.3)$ & & $110(8.3)$ & $109(8.3)$ & \\
\hline Body mass index & $29.9 \pm 6.6$ & $27.5 \pm 5.5$ & -0.372 & $28.6 \pm 5.8$ & $27.5 \pm 5.5$ & -0.205 \\
\hline \multicolumn{7}{|l|}{ Comorbidities } \\
\hline Charlson Comorbidity Index & $4.7 \pm 2.9$ & $6.9 \pm 2.9$ & 0.845 & $6.0 \pm 3.0$ & $6.9 \pm 2.9$ & 0.387 \\
\hline Diabetes mellitus & $28,617(65.4)$ & $945(70.7)$ & 0.149 & $959(72.2)$ & $940(70.7)$ & 0.000 \\
\hline Cerebrovascular disease & $13,347(30.5)$ & $865(64.8)$ & 0.970 & $835(62.8)$ & $857(64.5)$ & 0.232 \\
\hline Hypertension & $19,731(45.1)$ & $651(48.7)$ & 0.447 & $645(48.5)$ & $647(48.7)$ & 0.201 \\
\hline Myocardial infarction & $12,688(29.0)$ & $486(36.4)$ & 0.171 & $47(35.4)$ & $481(36.2)$ & 0.020 \\
\hline Congestive heart failure & $25,145(57.5)$ & $936(70.0)$ & 0.320 & $941(70.8)$ & $928(69.8)$ & 0.011 \\
\hline Peripheral vascular disease & $17,453(39.9)$ & $686(51.4)$ & 0.303 & $666(50.1)$ & $683(51.4)$ & 0.068 \\
\hline Paraplegia and hemiplegia & $1,517(3.5)$ & $140(10.5)$ & 0.431 & $128(9.6)$ & $134(10.1)$ & 0.191 \\
\hline Chronic pulmonary disease & $19,458(44.5)$ & $760(56.9)$ & 0.247 & $753(56.7)$ & $755(56.8)$ & 0.019 \\
\hline Connective tissue disease & $1,998(4.6)$ & $87(6.5)$ & 0.119 & $89(6.7)$ & $86(6.5)$ & -0.040 \\
\hline Liver disease & $5,146(11.8)$ & $171(12.8)$ & -0.137 & $167(12.5)$ & $167(12.5)$ & -0.113 \\
\hline Peptic ulcer disease & $3,636(8.3)$ & $172(12.9)$ & -0.203 & $181(13.6)$ & $169(12.7)$ & -0.397 \\
\hline Malignancies & $11,139(25.5)$ & $400(29.9)$ & 0.133 & $423(31.8)$ & $396(29.8)$ & 0.084 \\
\hline AIDS/HIV & $369(0.8)$ & $11(0.8)$ & -0.014 & $9(0.7)$ & $11(0.8)$ & -0.076 \\
\hline \multicolumn{7}{|l|}{ Medications } \\
\hline ACEIs/ARBs & $15,280(34.9)$ & $450(33.7)$ & 0.029 & $448(33.7)$ & $447(33.6)$ & -0.053 \\
\hline$\beta$-Blockers & $23,182(53.0)$ & $762(57.0)$ & 0.164 & $730(54.9)$ & $758(57.0)$ & 0.158 \\
\hline Calcium channel blockers & $20,320(46.4)$ & $659(49.3)$ & 0.127 & $637(47.9)$ & $653(49.1)$ & 0.076 \\
\hline Diuretics & $24,440(55.8)$ & 727 (54.4) & 0.060 & $692(52.1)$ & $722(54.3)$ & 0.121 \\
\hline Vasodilators & $1,288(2.9)$ & $42(3.1)$ & 0.112 & $33(2.5)$ & $42(3.2)$ & 0.166 \\
\hline Statins & $20,324(46.5)$ & $639(47.8)$ & 0.090 & $605(45.5)$ & $636(47.8)$ & 0.037 \\
\hline Vitamin D analogs & $9,618(21.9)$ & $273(20.4)$ & 0.026 & $243(18.3)$ & $270(20.3)$ & 0.143 \\
\hline ESAs & $7,319(16.7)$ & $240(17.9)$ & 0.168 & $244(18.3)$ & $237(17.8)$ & -0.037 \\
\hline Phosphate binders & $8,466(19.4)$ & $206(15.4)$ & -0.011 & $207(15.5)$ & $205(15.4)$ & 0.016 \\
\hline Bicarbonates & $4,566(10.4)$ & $111(8.3)$ & -0.080 & $105(7.9)$ & $109(8.2)$ & -0.042 \\
\hline \multicolumn{7}{|l|}{ Laboratory parameters } \\
\hline Serum albumin, g/dL & $3.3 \pm 0.6$ & $3.2 \pm 0.7$ & -0.112 & $3.2 \pm 0.6$ & $3.2 \pm 0.7$ & -0.048 \\
\hline Blood hemoglobin, g/dL & $10.3 \pm 1.5$ & $10.3 \pm 1.5$ & 0.052 & $10.2 \pm 1.5$ & $10.3 \pm 1.5$ & 0.097 \\
\hline eGFR, $\mathrm{mL} / \mathrm{min} / 1.73 \mathrm{~m}^{2}$ & $14.8 \pm 9.6$ & $15.4 \pm 8.5$ & 0.115 & $14.7 \pm 8.2$ & $15.3 \pm 8.5$ & 0.175 \\
\hline Serum creatinine, $\mathrm{mg} / \mathrm{dL}$ & $4.6 \pm 2.4$ & $4.0 \pm 1.7$ & -0.350 & $4.2 \pm 1.8$ & $4.0 \pm 1.7$ & -0.226 \\
\hline Blood urea nitrogen, $\mathrm{mg} / \mathrm{dL}$ & $61.1 \pm 23.2$ & $57.0 \pm 21.3$ & -0.225 & $60.3 \pm 22.4$ & $56.9 \pm 21.3$ & -0.207 \\
\hline Serum phosphorus, mg/dL & $5.1 \pm 1.3$ & $4.8 \pm 1.1$ & -0.278 & $4.9 \pm 1.2$ & $4.8 \pm 1.1$ & -0.124 \\
\hline Serum calcium, mg/dL & $8.8 \pm 0.7$ & $8.8 \pm 0.7$ & -0.006 & $8.8 \pm 0.7$ & $8.8 \pm 0.7$ & 0.018 \\
\hline Serum ALP, U/dL & $98.4 \pm 65.1$ & $94.0 \pm 50.7$ & -0.187 & $94.5 \pm 47.8$ & $93.9 \pm 50.8$ & -0.123 \\
\hline Serum AST, U/L & $26.1 \pm 39.0$ & $24.6 \pm 23.2$ & -0.038 & $22.9 \pm 17.0$ & $24.6 \pm 23.2$ & 0.148 \\
\hline Serum ALT, U/L & $24.0 \pm 33.9$ & $21.7 \pm 15.7$ & -0.093 & $20.2 \pm 12.9$ & $21.6 \pm 15.7$ & 0.146 \\
\hline Serum total cholesterol, mg/dL & $155.2 \pm 50.2$ & $149.5 \pm 43.1$ & -0.115 & $151.7 \pm 47.3$ & $149.5 \pm 43.1$ & -0.076 \\
\hline White blood cells, $\times 10^{9} / \mathrm{L}$ & $7.8 \pm 3.1$ & $7.9 \pm 3.2$ & -0.001 & $7.5 \pm 2.6$ & $7.9 \pm 3.2$ & 0.074 \\
\hline Serum bicarbonate, $\mathrm{mEq} / \mathrm{L}$ & $23.1 \pm 4.0$ & $23.4 \pm 3.8$ & 0.105 & $23.5 \pm 4.1$ & $23.3 \pm 3.8$ & 0.044 \\
\hline Serum potassium, $\mathrm{mmol} / \mathrm{L}$ & $4.5 \pm 0.6$ & $4.5 \pm 0.5$ & -0.086 & $4.5 \pm 0.5$ & $4.5 \pm 0.5$ & -0.083 \\
\hline
\end{tabular}

Data are presented as $n(\%)$ or mean \pm standard deviation. All laboratory results were averaged over the 6-month prelude period. ACEIs, angiotensin-converting enzyme inhibitors; ALP, alkaline phosphatase; ALT, alanine aminotransferase; ARBs, angiotensin receptor blockers; AST, aspartate aminotransferase; eGFR, estimated glomerular filtration rate; ESAs, erythropoiesis-stimulating agents; N/A, not applicable; std. diff., standardized difference. 
Molnar et al.: Pre-ESRD Dementia and Post-ESRD Mortality in a Large Cohort of Incident Dialysis Patients

Table 2. Predictors of presence of dementia using logistic regression analysis in the entire cohort

\begin{tabular}{|c|c|c|}
\hline & OR & $95 \%$ CI of OR \\
\hline Age (+1 year) & 1.06 & $1.05-1.07$ \\
\hline Gender: female vs. male (ref.) & 1.32 & $1.01-1.72$ \\
\hline \multicolumn{3}{|l|}{ Race } \\
\hline White (ref.) & 1.00 & $\mathrm{~N} / \mathrm{A}$ \\
\hline African-American & 1.83 & $1.59-2.10$ \\
\hline Other & 1.10 & $0.73-1.65$ \\
\hline \multicolumn{3}{|l|}{ Marital status } \\
\hline Married (ref.) & 1.00 & $\mathrm{~N} / \mathrm{A}$ \\
\hline Divorced & 1.33 & $1.07-1.65$ \\
\hline Single & 1.28 & $1.08-1.53$ \\
\hline Widowed & 0.98 & $0.82-1.16$ \\
\hline Other & 0.86 & $0.69-1.08$ \\
\hline Presence of diabetes vs. absence of diabetes (ref.) & 1.27 & $1.12-1.44$ \\
\hline Presence of cerebrovascular disease vs. absence of cerebrovascular disease (ref.) & 3.26 & $2.88-3.69$ \\
\hline Presence of hypertension vs. absence of hypertension (ref.) & 0.94 & $0.84-1.05$ \\
\hline Presence of myocardial infarction vs. absence of myocardial infarction (ref.) & 0.93 & $0.82-1.05$ \\
\hline Presence of congestive heart failure vs. absence of congestive heart failure (ref.) & 1.11 & $0.97-1.27$ \\
\hline Presence of PVD vs. absence of PVD (ref.) & 0.99 & $0.88-1.11$ \\
\hline Presence of paraplegia and hemiplegia vs. absence of paraplegia and hemiplegia (ref.) & 1.85 & $1.53-2.27$ \\
\hline Presence of chronic lung disease vs. absence of chronic lung disease (ref.) & 1.22 & $1.09-1.38$ \\
\hline Presence of connective tissue disease vs. absence of connective tissue disease (ref.) & 1.11 & $0.88-1.39$ \\
\hline Presence of liver disease vs. absence of liver disease (ref.) & 1.18 & $0.99-1.40$ \\
\hline Presence of peptic ulcer disease vs. absence of peptic ulcer disease (ref.) & 1.16 & $0.98-1.37$ \\
\hline Presence of malignancy vs. absence of malignancy (ref.) & 0.94 & $0.83-1.06$ \\
\hline Presence of AIDS/HIV vs. absence of AIDS/HIV (ref.) & 1.83 & $0.98-3.42$ \\
\hline Presence of ACEI/ARB use vs. absence of ACEI/ARB use (ref.) & 0.97 & $0.85-1.10$ \\
\hline Presence of $\beta$-blocker use vs. absence of $\beta$-blocker use (ref.) & 1.32 & $1.15-1.53$ \\
\hline Presence of CCB use vs. absence of CCB use (ref.) & 1.18 & $1.04-1.35$ \\
\hline Presence of diuretic use vs. absence of diuretic use (ref.) & 0.76 & $0.66-0.88$ \\
\hline Presence of vasodilator use vs. absence of vasodilator use (ref.) & 1.17 & $0.84-1.61$ \\
\hline Presence of statin use vs. absence of statin use (ref.) & 0.93 & $0.81-1.06$ \\
\hline Presence of vitamin D analog use vs. absence of vitamin D analog use (ref.) & 0.87 & $0.75-1.01$ \\
\hline Presence of ESA use vs. absence of ESA use (ref.) & 1.25 & $1.06-1.47$ \\
\hline Presence of phosphate binder use vs. absence of phosphate binder use (ref.) & 0.89 & $0.75-1.06$ \\
\hline Presence of bicarbonate use vs. absence of bicarbonate use (ref.) & 0.86 & $0.69-1.06$ \\
\hline
\end{tabular}

ACEI, angiotensin-converting enzyme inhibitor; ARB, angiotensin receptor blocker; $\mathrm{CCB}$, calcium channel blocker; CI, confidence interval; ESA, erythropoiesis-stimulating agent; N/A, not applicable; OR, odds ratio; PVD, peripheral vascular disease.

plegia/hemiplegia, lung disease, rheumatic disease, peptic ulcer disease, HIV/AIDS, malignancy, liver disease, diabetes, hypertension), and medications at baseline (statins, vasodilators, renin-angiotensin-aldosterone system blockers, phosphate binders, erythropoiesis-stimulating agents, diuretics, calcium channel blockers, bicarbonates, $\beta$-blockers, active vitamin D). Finally, we performed sensitivity analyses in the entire cohort as well as by creating a new propensity-matched cohort with adjustment for emergency department visits (as proxy for falls) in the last year before ESRD initiation as well as use of aspirin, donepezil, and memantine at baseline.

The associations of dementia with outcomes were examined in subgroups of patients stratified by sex, age, race, marital status, and presence/absence of selected comorbidities. Potential interactions were formally tested including relevant interaction terms in the statistical models and using the Wald test.

Reported $p$ values were 2 -sided and reported as significant at $<0.05$ for all analyses. All analyses were conducted using Stata/MP version 14 (Stata Corp., College Station, TX, USA). The study was approved by the Institutional Review Boards of the Memphis and Long Beach VA Medical Centers, with exemption from informed consent. 
Fig. 2. Associations between presence of dementia and allcause mortality in the propensitymatched cohort during the first 6-month period.
Molnar et al.: Pre-ESRD Dementia and Post-ESRD Mortality in a Large Cohort of Incident Dialysis Patients

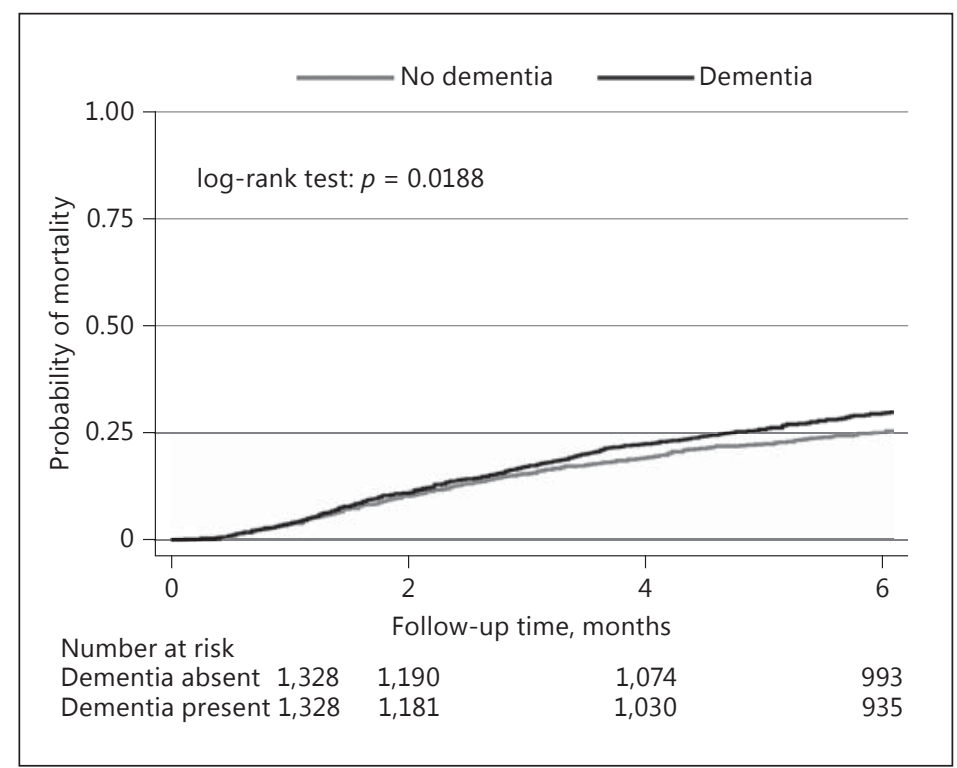

\section{Results}

\section{Baseline Characteristics}

The baseline characteristics of the overall cohort before and after propensity score matching and stratified by the presence of dementia are presented in Table 1 . In the entire cohort, the overall mean \pm SD age at baseline was $72 \pm 11$ years, $95 \%$ were male, $23 \%$ were African-American, and $66 \%$ were diabetic. We identified $1,336(3.0 \%)$ patients with an ICD-9-CM code for dementia. The mean \pm SD 6-month prelude averaged eGFR level was 14.8 $\pm 9.5 \mathrm{~mL} / \mathrm{min} / 1.73 \mathrm{~m}^{2}$. The median and interquartile range (IQR) between the date of dialysis initiation and the date of first detected and last detected dementia diagnosis were 634 (1731,362) days and 315 (58-860) days, respectively.

In the original cohort $(n=45,076)$, patients with dementia were slightly older and had more comorbidities: higher prevalence of cerebrovascular disease, diabetes mellitus, congestive heart failure, peripheral artery disease, paraplegia, hemiplegia, and chronic pulmonary disease. In our adjusted logistic regression model, which served for calculating propensity scores for odds of dementia diagnoses prior to RRT transition, female gender, African-American race, and being divorced or single, most of the comorbidities (such as diabetes, cerebrovascular disease, paraplegia/hemiplegia, chronic lung disease) and certain medications ( $\beta$-blockers, calcium channel blockers, and erythropoiesis-stimulating agents) were associated with a higher risk of dementia (Table 2). After propensity score matching most of these differences disappeared and the baseline characteristics of patients with and without dementia were balanced (Table 1). In the propensity-matched cohort, the overall mean \pm SD age at baseline was $77 \pm 9$ years, $95 \%$ were male, $24 \%$ were African-American, and $71 \%$ were diabetic.

Association of Pre-ESRD Presence of Dementia with Post-ESRD All-Cause Mortality in the First 6 Months after Dialysis Initiation

In the entire cohort, there were 8,080 (18.5\%) deaths (mortality rate, 412 ; $95 \%$ confidence interval [CI], 403-421/1,000 patient-years) in the dementia-negative group, and 396 (29.6\%) deaths (mortality rate, 708; 95\% CI, 642-782/1,000 patient-years) in the dementiapositive group during the first 6 months after dialysis initiation. In the propensity-matched cohort, there were 736 (27.7\%) deaths (mortality rate, 656; 95\% CI, 610-705/1,000 patient- 
Dementia

Cognitive Disorders

Fig. 3. Adjusted hazard ratios (95\% CIs) of presence of dementia for all-cause mortality in the first 6 months after dialysis initiation in selected subgroups. CHF, congestive heart failure; CI, confidence interval; CVD, cerebrovascular disease; HR, hazard ratio.

Fig. 4. Associations between presence of dementia and allcause mortality in the entire cohort during the first 6-month period.

\begin{tabular}{l|l}
\hline \multicolumn{2}{l|}{ Dement Geriatr Cogn Disord 2017;43:281-293 } \\
\hline DOI: 10.1159/000471761 & $\begin{array}{l}\text { C 2017 S. Karger AG, Basel } \\
\text { www.karger.com/dem }\end{array}$ \\
\hline
\end{tabular}

Molnar et al.: Pre-ESRD Dementia and Post-ESRD Mortality in a Large Cohort of Incident Dialysis Patients
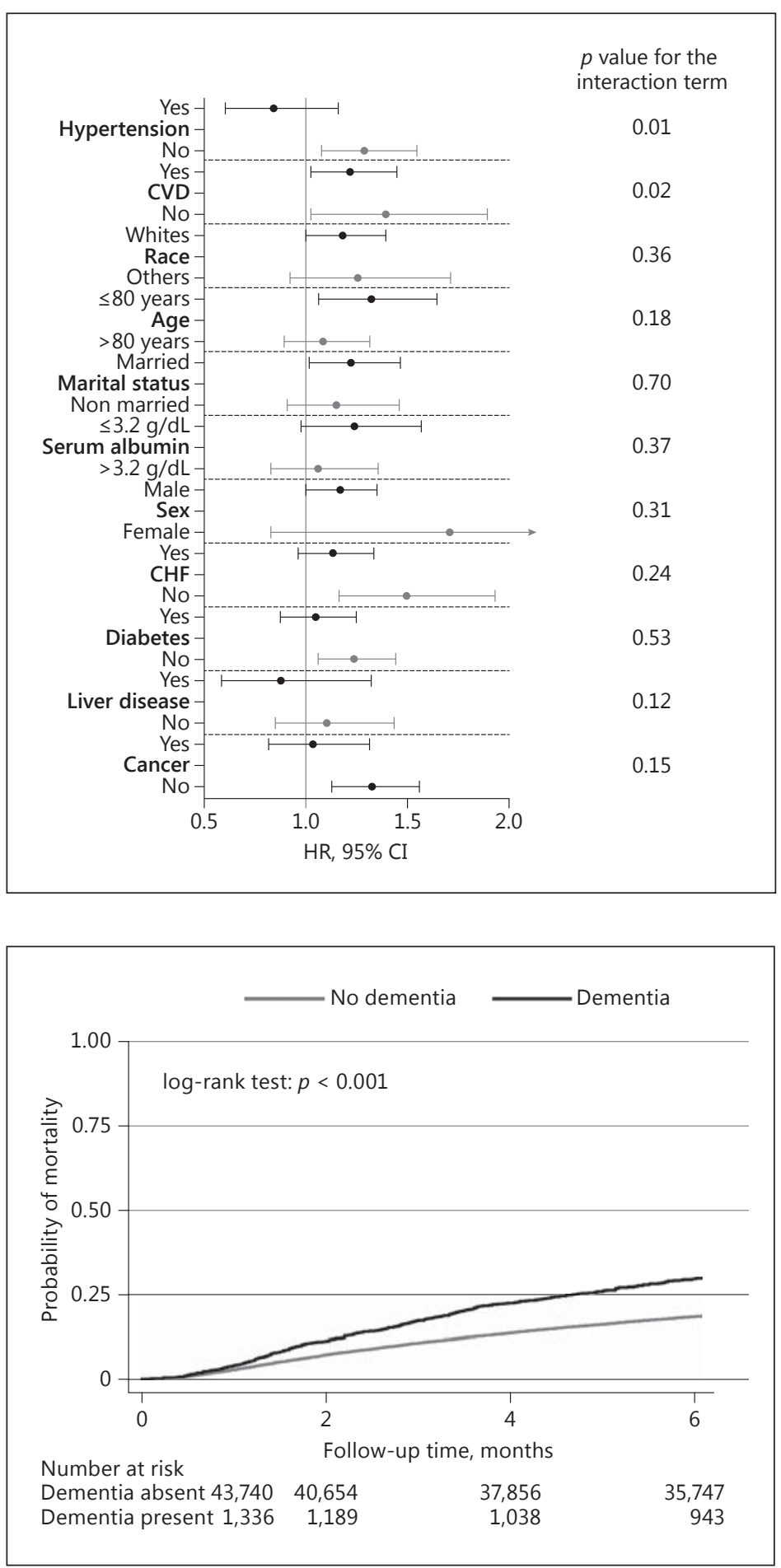

years) during the first 6 months after dialysis initiation; of these deaths, 340 (25.6\%; mortality rate, 599; 95\% CI, 538-666/1,000 patient-years) occurred in the dementia-negative group, and 396 (29.8\%; mortality rate, 714; 95\% CI, 645-787/1,000 patient-years) in the dementiapositive group. Figure 2 shows the associations between presence of dementia and all-cause mortality in the propensity-matched cohort during the first 6-month period. Presence of dementia was associated with higher risk of all-cause mortality (hazard ratio [HR], 1.19; 95\% 
Molnar et al.: Pre-ESRD Dementia and Post-ESRD Mortality in a Large Cohort of Incident Dialysis Patients

Table 3. Association of presence of dementia and all-cause mortality in different follow-up periods in the propensity-matched cohort

\begin{tabular}{|c|c|c|c|c|c|c|}
\hline & \multicolumn{2}{|l|}{ 6-month survival } & \multicolumn{2}{|c|}{ 12-month survival } & \multicolumn{2}{|c|}{ Survival during the entire follow-up } \\
\hline & $\begin{array}{l}\text { absence of } \\
\text { dementia }\end{array}$ & $\begin{array}{l}\text { presence of } \\
\text { dementia }\end{array}$ & $\begin{array}{l}\text { absence of } \\
\text { dementia }\end{array}$ & $\begin{array}{l}\text { presence of } \\
\text { dementia }\end{array}$ & $\begin{array}{l}\text { absence of } \\
\text { dementia }\end{array}$ & $\begin{array}{l}\text { presence of } \\
\text { dementia }\end{array}$ \\
\hline Events & 340 & 396 & 503 & 606 & 904 & 1,019 \\
\hline Crude incident rate & $599(538-666)$ & $714(645-787)$ & $494(453-539)$ & $630(582-682)$ & $399(374-426)$ & $535(503-568)$ \\
\hline Hazard ratio $(95 \% \mathrm{CI})$ & 1 (ref.) & $1.19(1.03-1.37)$ & 1 (ref.) & $1.26(1.12-1.42)$ & 1 (ref.) & $1.30(1.19-1.42)$ \\
\hline
\end{tabular}

Data are presented as number or hazard ratio $(95 \% \mathrm{CI})$ unless otherwise specified. The crude incident rate presented in per 1,000 patient-years. CI, confidence interval.

CI, 1.03-1.37) compared to dementia-free patients (Table 3). Similar results were found in almost all subgroups, and only presence of cerebrovascular disease and hypertension were significant effect modifiers of the dementia-mortality association (Fig. 3). Qualitatively similar results (HR, 1.29; 95\% CI, 1.16-1.44) were found when we additionally adjusted for eGFR, serum albumin, and blood hemoglobin in sensitivity analysis restricted to a limited subset of our propensity-matched cohort $(n=1,840)$. In analyses including the overall population cohort (Fig. 4), patients with dementia also had significantly higher mortality rates and qualitatively similar results (HR, 1.25; 95\% CI, 1.12-1.38) in adjusted Cox regression models (online suppl. Table 1; see www.karger.com/doi/10.1159/000471761 for all online suppl. material). Finally, qualitatively similar results were found after we adjusted for emergency department visits (as proxy for falls) in the last year before ESRD initiation as well as use of aspirin, donepezil, and memantine at baseline (not shown).

Association of Pre-ESRD Dementia with Post-ESRD All-Cause Mortality in the Entire

Follow-Up Period and the First 12 Months after Dialysis Initiation

Over the entire follow-up period, the median follow-up time was 16.2 months (IQR, 5.1-28.5 months). In the entire cohort, there were 24,877 (56.9\%) deaths (mortality rate, 281; 95\% CI, 278-285/1,000 patient-years) in the dementia-negative group, and 1,024 (76.6\%) deaths (mortality rate, 534; 95\% CI, 502-567/1,000 patient-years) in the dementiapositive group over the entire follow-up period. In the propensity-matched cohort, there were 1,923 (72.4\%) deaths (mortality rate, 461; 95\% CI, 441-482/1,000 patient-years) during this time, of which 904 (68.1\%) deaths (mortality rate, 399; 95\% CI, 374-426/1,000 patient-years) were in the dementia-negative group, and 1,019 (76.7\%) deaths (mortality rate, 535; 95\% CI, 503-568/1,000 patient-years) were in the dementia-positive group. Presence of dementia was associated with a higher risk of all-cause mortality (HR, 1.30; 95\% CI, 1.19-1.42) compared to dementia-free patients in the propensity-matched cohort during the entire follow-up period (Table 3). Similar results were found in almost all subgroups, and only presence of cerebrovascular disease and congestive heart failure were significant effect modifiers on these dementia-mortality associations (online suppl. Fig. 1).

Qualitatively similar associations were found in the analysis for 12-month follow-up in the overall propensity-matched cohort (HR, 1.26; 95\% CI, 1.12-1.42) (Table 3) as well as in the subgroup analysis (online suppl. Fig. 2). In the entire cohort, only 54 and $11 \%$ of the patients with dementia were still alive 1 and 5 years after dialysis initiation, respectively. Sensitivity analyses evaluating dementia-mortality associations over these follow-up periods for the entire cohort population showed similar results (online suppl. Table 1). Finally, qualitatively similar results were found after we adjusted for emergency department visits (as proxy for falls) in the last year before ESRD initiation as well as use of aspirin, donepezil, and memantine at baseline (not shown). 
Molnar et al.: Pre-ESRD Dementia and Post-ESRD Mortality in a Large Cohort of Incident Dialysis Patients

\section{Discussion}

In this large national cohort of US veterans with late-stage NDD-CKD transitioning to dialysis, we found a modest association between pre-ESRD dementia and early all-cause mortality following dialysis initiation, independent of demographics, comorbidities, and medications. These associations were most accentuated for mortality experienced in the immediate 6-12 months following ESRD transition.

Our study examined the association of dementia diagnosed in the pre-ESRD period with mortality occurring immediately after transition to dialysis. Previous studies assessing outcomes associated with dementia in dialysis patients examined prevalent cohorts, in which dementia might have developed or worsened after the initiation of dialysis therapy $[12,14$, 15]. Dementia present prior to dialysis transition can be an important factor in deciding whether or not a patient pursues RRT or chooses conservative management of ESRD; therefore, it is important to examine what outcomes dementia may portend in a patient opting for dialysis. Our findings of high early post-transition all-cause mortality associated with pre-ESRD dementia may add useful information to help such decision-making, although further studies are needed to examine patient-centered outcomes such as quality of life, fracture rates, or hospitalizations.

Presence of dementia before ESRD was associated with a 19\% higher risk of post-ESRD all-cause mortality during the first 6 months after ESRD initiation in our propensity-matched cohort. The potential underlying mechanisms linking dementia to higher risk of post-ESRD mortality are likely to be multifactorial and may be related to factors including poor treatment and medication adherence, comorbidity, worse nutritional status, and dementia itself [22]. Hemodialysis typically requires strict adherence to treatment regimens, which likely will be impaired with cognitive defects [23]. Poor functional and nutritional status may also predispose dementia patients on hemodialysis to an increased risk of mortality. In a large retrospective cohort study by Rakowski et al. [15], hemodialysis patients with dementia had a lower body mass index and lower albumin levels. Moreover, a higher prevalence of coronary artery disease and congestive heart failure in dementia patients was also noted in this cohort, which can lead to an increased morbidity burden [15]. However, in our study the presence of dementia was associated with a higher risk of death even after adjusting for these potential confounders. Additionally, dementia is a well-known independent risk factor for hip fractures associated with a high rate of morbidity and mortality [24, 25], and also shows strong association with depression, which is another predictor of survival [26, 27]. Finally, the morbidity connected with a diagnosis of dementia is also associated with large amounts of direct as well as indirect health care costs, while dementia patients typically fall in a lower income group [28]. Patients with lower income are more likely to postpone medical care because of obligatory copayments and lack of insight into the illness. These people are also more likely nonadherent, which can contribute to the increased mortality risk [8]. In a large prospective cohort of roughly 15,000 patients, low socioeconomic status was found to be contributing to the increased risk of mortality in patients with dementia [29]. Finally, the caregivers' perspective is not to be neglected. The medical diagnosis of dementia continues to be marred by social stigma. Nearly half of the participants in a Japanese study report being ashamed of a family member being diagnosed with dementia [30]. Poor mental health literacy among the public regarding dementia juxtaposed with negative public attitude serves as a major deterrent to seek timely help from a medical professional [31], which can ultimately lead to more severe adverse outcomes.

It is important to emphasize that more than $25 \%$ of patients with dementia died within 6 months of the initiation of dialysis, while almost $50 \%$ of them did not survive the first year. We did not analyze the rate of hospitalizations during this period, and we did not have data 
Molnar et al.: Pre-ESRD Dementia and Post-ESRD Mortality in a Large Cohort of Incident Dialysis Patients

about quality of life available for analysis. Regardless, the higher mortality rate observed in dementia patients indicates that alternative therapies such as conservative management might be desirable in this selected population; however, further studies assessing patientcentered outcomes such as quality of life after transition to dialysis, and comparing mortality rates in patients who transition to dialysis compared to those who prefer conservative care are needed. Our data may support the recommendations of the Renal Physicians Association (RPA), which suggest that patients with advanced dementia be advised against initiation of dialysis, particularly when they are unable to cooperate with the dialysis process [10, 32]. Supporting this recommendation, the Dialysis Advisory Group of the American Society of Nephrology suggested that dialysis patients with a life expectancy $<1$ year may desire a palliative approach to dialysis care, which focuses on aligning patient treatment with patients' informed preferences [9]. The RPA emphasizes the need for establishing a strong therapeutic relationship and open communication to facilitate shared decision-making [32]. However, the "first, do not harm" ethical principle should have a significant role in this shared decisionmaking by patients, caregivers, and physicians. Further research is needed to identify the most important factors which improve the quality of life of patients who decide to choose conservative treatment for ESRD.

Our study is notable for its large sample size and event numbers and for likely being representative of veterans who received care in the VA system across the entire USA. It also has several limitations that need to be acknowledged. First, since this was an observational study, only associations, but no cause-effect relationships, can be established. Second, most of our patients were male US veterans; hence, the results may not be generalizable to women or other patient populations, in particular those outside the USA. Third, our study is also limited by the use of diagnostic codes to define dementia. The sensitivity and specificity of these codes in ascertaining these conditions is not known, and it is likely lower than prospective adjudication of clinical diagnoses [13]. However, the significant correlates of baseline dementia in our study were similar to those found in previous studies [15]. In addition, we analyzed only all-cause mortality, while other outcomes such as quality of life and frequency of hospitalization would be important factors to examine and consider in the clinical decisionmaking for dementia patients. Finally, due to the observational nature of our study, adjusted analyses were limited to confounders measured and available in our cohort, and therefore our study may be limited by potential residual confounding from unmeasured confounders such as proteinuria and level of education.

In conclusion, in this large national cohort of US veterans with late-stage NDD-CKD transitioning to dialysis, we found an independent association between pre-ESRD diagnosis of dementia and all-cause mortality early after dialysis initiation. Clinical studies are warranted to compare survival and quality of life of dialysis treatment versus conservative treatment in patients with dementia.

\section{Acknowledgments}

C.P. Kovesdy and K. Kalantar-Zadeh are employees of the Department of Veterans Affairs. The interpretation and reporting of these data are the responsibility of the authors and should in no way be seen as official policy or interpretation of the Department of Veterans Affairs or the US government. The results of this paper have not been published previously in whole or part.

This study was supported by grant 5U01DK102163 from the National Institutes of Health (NIH) to K. Kalantar-Zadeh and C.P. Kovesdy, and by resources from the US Department of Veterans Affairs. The data reported here were supplied in part by the USRDS. Support for VA/CMS data was provided by the Department of Veterans Affairs, Veterans Health Administration, Office of Research and Development, Health Services Research and Development, VA Information Resource Center (Project Numbers SDR 02-237 and 98-004). 
Dementia

Cognitive Disorders

\begin{tabular}{l|l}
\hline \multicolumn{2}{l|}{ Dement Geriatr Cogn Disord 2017:43:281-293 } \\
\hline DOI: 10.1159/000471761 & $\begin{array}{l}\text { C 2017 S. Karger AG, Basel } \\
\text { www.karger.com/dem }\end{array}$ \\
\hline
\end{tabular}

Molnar et al.: Pre-ESRD Dementia and Post-ESRD Mortality in a Large Cohort of Incident Dialysis Patients

\section{Disclosure Statement}

None of the authors have any relevant conflicts of interest. The funders of this study had no role in the study design, the collection, analysis, and interpretation of data, the writing of the paper, or the decision to submit the paper for publication.

\section{Author Contributions}

Study concept and design: all authors. Acquisition of data: M.Z. Molnar, K. Sumida, P.K. Potukuchi, J.L. Lu, and C.P. Kovesdy. Analysis and interpretation of data: M.Z. Molnar and C.P. Kovesdy. Drafting of the manuscript and approval of the final version: M.Z. Molnar, E. Streja, and C.P. Kovesdy. Critical revision of the manuscript for important intellectual content and approval of the final version: all authors. All authors contributed important intellectual content during manuscript drafting or revision and accept accountability for the overall work by ensuring that questions pertaining to the accuracy or integrity of any portion of the work were appropriately investigated and resolved. C.P. Kovesdy and M.Z. Molnar take responsibility that this study has been reported honestly, accurately, and transparently, that no important aspects of the study have been omitted, and that any discrepancies from the study as planned (and, if relevant, registered) have been explained.

\section{References}

1 Saran R, Li Y, Robinson B, Ayanian J, Balkrishnan R, Bragg-Gresham J, Chen JT, Cope E, Gipson D, He K, Herman W, Heung M, Hirth RA, Jacobsen SS, Kalantar-Zadeh K, Kovesdy CP, Leichtman AB, Lu Y, Molnar MZ, Morgenstern H, Nallamothu B, O’Hare AM, Pisoni R, Plattner B, Port FK, Rao P, Rhee CM, Schaubel DE, Selewski DT, Shahinian V, Sim JJ, Song P, Streja E, Kurella Tamura M, Tentori F, Eggers PW, Agodoa LY, Abbott KC: US Renal Data System 2014 Annual Data Report: Epidemiology of Kidney Disease in the United States. Am J Kidney Dis 2015;66(1 suppl 1):Svii, S1-S305.

2 Saran R, Li Y, Robinson B, Abbott KC, Agodoa LY, Ayanian J, Bragg-Gresham J, Balkrishnan R, Chen JL, Cope E, Eggers PW, Gillen D, Gipson D, Hailpern SM, Hall YN, He K, Herman W, Heung M, Hirth RA, Hutton D, Jacobsen SJ, Kalantar-Zadeh K, Kovesdy CP, Lu Y, Molnar MZ, Morgenstern H, Nallamothu B, Nguyen DV, O'Hare AM, Plattner B, Pisoni R, Port FK, Rao P, Rhee CM, Sakhuja A, Schaubel DE, Selewski DT, Shahinian V, Sim JJ, Song P, Streja E, Kurella Tamura M, Tentori F, White S, Woodside K, Hirth RA: US Renal Data System 2015 Annual Data Report: Epidemiology of Kidney Disease in the United States. Am J Kidney Dis 2016;67(3 suppl 1):Svii, S1-S305.

3 Bradbury BD, Fissell RB, Albert JM, Anthony MS, Critchlow CW, Pisoni RL, Port FK, Gillespie BW: Predictors of early mortality among incident US hemodialysis patients in the Dialysis Outcomes and Practice Patterns Study (DOPPS). Clin J Am Soc Nephrol 2007;2:89-99.

4 Soucie JM, McClellan WM: Early death in dialysis patients: risk factors and impact on incidence and mortality rates. J Am Soc Nephrol 1996;7:2169-2175.

5 Sumida K, Molnar MZ, Potukuchi PK, Thomas F, Lu JL, Ravel VA, Soohoo M, Rhee CM, Streja E, Yamagata K, Kalantar-Zadeh K, Kovesdy CP: Association between vascular access creation and deceleration of estimated glomerular filtration rate decline in late-stage chronic kidney disease patients transitioning to end-stage renal disease. Nephrol Dial Transplant 2016, Epub ahead of print.

6 Jassal SV, Karaboyas A, Comment LA, Bieber BA, Morgenstern H, Sen A, Gillespie BW, De Sequera P, Marshall MR, Fukuhara S, Robinson BM, Pisoni RL, Tentori F: Functional dependence and mortality in the international Dialysis Outcomes and Practice Patterns Study (DOPPS). Am J Kidney Dis 2016;67:283-292.

7 Sumida K, Molnar MZ, Potukuchi PK, Thomas F, Lu JL, Jing J, Ravel VA, Soohoo M, Rhee CM, Streja E, KalantarZadeh K, Kovesdy CP: Association of slopes of estimated glomerular filtration rate with post-end-stage renal disease mortality in patients with advanced chronic kidney disease transitioning to dialysis. Mayo Clin Proc 2016;91:196-207.

8 Molnar MZ, Gosmanova EO, Sumida K, Potukuchi PK, Lu JL, Jing J, Ravel VA, Soohoo M, Rhee CM, Streja E, Kalantar-Zadeh K, Kovesdy CP: Predialysis cardiovascular disease medication adherence and mortality after transition to dialysis. Am J Kidney Dis 2016;68:609-618.

9 Grubbs V, Moss AH, Cohen LM, Fischer MJ, Germain MJ, Jassal SV, Perl J, Weiner DE, Mehrotra R; Dialysis Advisory Group of the American Society of Nephrology: A palliative approach to dialysis care: a patientcentered transition to the end of life. Clin J Am Soc Nephrol 2014;9:2203-2209.

10 Ying I, Levitt Z, Jassal SV: Should an elderly patient with stage V CKD and dementia be started on dialysis? Clin J Am Soc Nephrol 2014;9:971-977.

11 Helvik AS, Engedal K, Benth JS, Selbaek G: Prevalence and severity of dementia in nursing home residents. Dement Geriatr Cogn Disord 2015;40:166-177. 
Molnar et al.: Pre-ESRD Dementia and Post-ESRD Mortality in a Large Cohort of Incident Dialysis Patients

12 Raphael KL, Wei G, Greene T, Baird BC, Beddhu S: Cognitive function and the risk of death in chronic kidney disease. Am J Nephrol 2012;35:49-57.

13 Murray AM, Tupper DE, Knopman DS, Gilbertson DT, Pederson SL, Li S, Smith GE, Hochhalter AK, Collins AJ, Kane RL: Cognitive impairment in hemodialysis patients is common. Neurology 2006;67:216-223.

14 Kurella M, Mapes DL, Port FK, Chertow GM: Correlates and outcomes of dementia among dialysis patients: the Dialysis Outcomes and Practice Patterns Study. Nephrol Dial Transplant 2006;21:2543-2548.

15 Rakowski DA, Caillard S, Agodoa LY, Abbott KC: Dementia as a predictor of mortality in dialysis patients. Clin J Am Soc Nephrol 2006;1:1000-1005.

16 United States Renal Data System, 2014 Annual Data Report: Epidemiology of Kidney Disease in the United States. Bethesda, MD, National Institutes of Health, National Institute of Diabetes and Digestive and Kidney Diseases, 2014.

17 US Department of Veterans Affairs: VIReC Research User Guide; VHA Medical SAS Inpatient Datasets FY20062007. Hines, IL, VA Information Resource Center, 2007.

18 VA Information Resource Center: VIReC Research User Guide: VHA Pharmacy Prescription Data, ed 2. Hines, IL, US Department of Veterans Affairs, Health Services Research and Development Service, VA Information Resource Center, 2008.

19 Kovesdy CP, Norris KC, Boulware LE, Lu JL, Ma JZ, Streja E, Molnar MZ, Kalantar-Zadeh K: Association of race with mortality and cardiovascular events in a large cohort of US veterans. Circulation 2015;132:1538-1548.

20 Kovesdy CP, Alrifai A, Gosmanova EO, Lu JL, Canada RB, Wall BM, Hung AM, Molnar MZ, Kalantar-Zadeh K: Age and outcomes associated with BP in patients with incident CKD. Clin J Am Soc Nephrol 2016;11:821-831.

21 Levey AS, Stevens LA, Schmid CH, Zhang YL, Castro AF 3rd, Feldman HI, Kusek JW, Eggers P, Van Lente F, Greene T, Coresh J; CKD-EPI (Chronic Kidney Disease Epidemiology Collaboration): A new equation to estimate glomerular filtration rate. Ann Intern Med 2009;150:604-612.

22 Garcia-Ptacek S, Farahmand B, Kareholt I, Religa D, Cuadrado ML, Eriksdotter M: Mortality risk after dementia diagnosis by dementia type and underlying factors: a cohort of 15,209 patients based on the Swedish Dementia Registry. J Alzheimers Dis 2014;41:467-477.

23 Griva K, Stygall J, Hankins M, Davenport A, Harrison M, Newman SP: Cognitive impairment and 7-year mortality in dialysis patients. Am J Kidney Dis 2010;56:693-703.

24 Maravic M, Ostertag A, Urena P, Cohen-Solal M: Dementia is a major risk factor for hip fractures in patients with chronic kidney disease. Osteoporos Int 2016;27:1665-1669.

25 Tolppanen AM, Taipale H, Tanskanen A, Tiihonen J, Hartikainen S: Comparison of predictors of hip fracture and mortality after hip fracture in community-dwellers with and without Alzheimer's disease - exposurematched cohort study. BMC Geriatr 2016;16:204.

26 Lara E, Koyanagi A, Domenech-Abella J, Miret M, Ayuso-Mateos JL, Haro JM: The impact of depression on the development of mild cognitive impairment over 3 years of follow-up: a population-based study. Dement Geriatr Cogn Disord 2017;43:155-169.

27 Chakrabarty T, Sepehry AA, Jacova C, Hsiung GY: The prevalence of depressive symptoms in frontotemporal dementia: a meta-analysis. Dement Geriatr Cogn Disord 2015;39:257-271.

28 Frahm-Falkenberg S, Ibsen R, Kjellberg J, Jennum P: Health, social and economic consequences of dementias: a comparative national cohort study. Eur J Neurol 2016;23:1400-1407.

29 van de Vorst IE, Koek HL, Stein CE, Bots ML, Vaartjes I: Socioeconomic disparities and mortality after a diagnosis of dementia: results from a nationwide registry linkage study. Am J Epidemiol 2016;184:219-226.

30 Aihara Y, Kato H, Sugiyama T, Ishi K, Goto Y: Public attitudes towards people living with dementia: a crosssectional study in urban Japan (innovative practice). Dementia (London) 2016, Epub ahead of print.

31 Picco L, Abdin E, Pang S, Vaingankar JA, Jeyagurunathan A, Chong SA, Subramaniam M: Association between recognition and help-seeking preferences and stigma towards people with mental illness. Epidemiol Psychiatr Sci 2016, Epub ahead of print.

32 Renal Physicians Association: Shared decision-making in the appropriate initiation of and withdrawal from dialysis: clinical practice guideline. Rockville, MD, Renal Physicians Association, 2010. 